\title{
QoS Aware Optimal Confederation based Radio Resource Management Scheme for LTE Networks
}

\author{
T. Ganga Prasad, MSS. Rukmini
}

\begin{abstract}
In this paper proposed for future generation Long Term Evolution (LTE) networks a radio resource management using QoS with aware QOC-RRM method. In QOC-RRM scheme we present the hybrid Recurrent Deep Neural Network (RDNN) technique to differentiate the operators by priority wise based on multiple constraints and it control the allocated resource bybase stations. For routing share queuing criterion data with other schaotic weed optimization (CWO) algorithm are proposed. Once information received each $B S$ schedules the resources for priority user first. The proposed QOC-RRM scheme is implemented in Network Simulator (NS3) tool and performance can better than conventional RRM schemes in terms of minimum date rate requirement, maximum number of active users and utilization of the radio spectrums.
\end{abstract}

Keywords: Quality of Service, Radio Resource Management, Long Term Evolution, Recurrent Deep Neural Network, chaotic weed optimization.

\section{INTRODUCTION}

LTE is the following significant advance in Universal Mobile Telecommunications System (UMTS) and will be presented in third Generation Partnership Project (3GPP). The design depends on an advancement of the current GSM/WCDMA center system, with improved activities and smooth, cost-productive sending. As the successor to UMTS, LTE should make transmissions conceivable at information rates of more than $100 \mathrm{Megabit} / \mathrm{s}$ in the downlink and more than $50 \mathrm{Megabit} / \mathrm{s}$ in the uplink just as diminish inertness for bundle transmissions. LTE bolster data transfer capacities of up to $20 \mathrm{MHz}$. Now a day's most of the cellular radio communications systems are depends upon the LTE techniques. These techniques consist of macro and various numbers of underlying small cells opposing for the similar radio resource [1]. This is because of various resource requirements and due to enlarging the users, the radio resource is charming rare. To overcome the demands for higher data rates with partial resource is becoming a dynamic issue for the developing fourth generation (4G) network [2]. Quality of service (QoS) discuss about the complete performance of the network.To reach the essential QoS level by handling the network parameter [3].On/off chance that the system essential Voice over IP (VoIP), and IPTV [4][5].

Revised Manuscript Received on December 15, 2019.

Mr.T.Ganga Prasad ,Research scholor,ECE,VFSTR,

Prasad4_1@rediffmail.com.

Mrs. Dr.MSS.Rukmini, Professor,ECE, VFSTR,Vignan University mssrukmini@gmail.com
The radio resource management (RRM) holds the power consumption of every sector in each cell. These cells are calculated depends upon the output of the antenna adjustment module. And SINR also calculated depends on the channels model, interference from the base adjacent BSs and transmission power of the BS. The limit of every user and the entire system is determined dependent on the power and SINR Recently, a few RRM methods have proposed for upcoming generation networks. The two D2D resource allotment protocols [6] utilized and the system efficiencies are evaluated to demonstrate the potential execution degradation when UCQ issue is not mentioned. A dynamic resource allocation algorithm [7] is utilized for the downlink transmission which includes resource part, modulation, component carriers, and coding plans and frequency barriers with overall consideration. QoS-aware resource management process is utilized for multimedia traffic report system over LTE-A (QoS-MTRS) [8] and it plans resource depends on the significance degrees of different traffic information. H-PSSS scheme [9] is utilized to diminish power utilization and improve spectrum productivity according to change in traffic load and channel conditions. The plan considers UE channel conditions, bandwidth requirements, and QoS. DiRAT [10] is scattered RRM scheme for D2D communication underlying cellular systems. D2D nodes locally select their radio resource from a pool made by the cellular network so as to control the interference produced to the essential cellular user. DiRAT incorporates a control mechanism to guarantee that the client QoS necessities are fulfilled. An on-the-fly RRS scheme [11] is presented for mobile foundations and gives mobile devices with the opportunity to get to all accessible radio resource around them. The new confederation idea [12] is resolved the individual base stations can assume control for their dispensed resource. LTE is a promising innovation to be connected for various applications because of its high infiltration, high information rate, reliability, and QoS support. Because of the obstruction experienced by the users, the forceful frequency reuse generally happened which cause the poor capacity at the cell edge. It likewise expands the accessible transmission data transfer capacity and planning a flexibility in uplink and presents extra power back-off for the power enhancer in the user equipment with nontouching resource distribution.

Our contributions: In this paper, we proposed quality of service aware optimal confederation based radio resource management (QOC-RRM) scheme so that individual base stations can take control of their allocated resource. In this scheme we introduced the hybrid recurrent deep neural network (RDNN) and chaotic weed optimization (CWO) algorithms. 
Related Works : In this section, we are going to discuss about the literature review ,David et al. have examined about an expository model for assessing SFR arrangements dependent on the spatial Poisson point process. This proposed structure demonstrates the effect of cross-level obstruction, illuminates determination regarding key FFR parameters in open access [13].

Park et al. examines at a symmetrical arbitrary beam framing based cross tier interference reduction schemefor two-tier femtocell systems [14]. Jeffrey et al. proposed the explanations behind this boundless distrust, and talk about how present and future patterns will build the requirement for and feasibility of multi-user collectors for both the uplink, where numerous offbeat clients will be at the same time distinguished [15]. Dong et al. present the characteristics of an optimal allocation of power and sub channel in dense environments. These authors prove that a special form of power allocation, which is called binary power allocation, in which only one transmitter transmits a signal for each sub channel, performs better than power allocation by conventional schemes [16]. Lee et al. presented a wireless scheduling algorithm based on the cumulative distribution function (CDF) and its simple modification that limits the maximum starving time. This cdf-based scheduling (CS) algorithm selects the user for transmission based on the cdf of user rates [17]. Raoet et al. developed an analytical framework to investigate heterogeneous partial feedback in a general OFDMA-based heterogeneous multi-cell employing the best-M partial feedback strategy [18]. Chandrasekhar et al. analyzed an optimum decentralized spectrum allocation policy for twotier networks that employ frequency division multiple access (including OFDMA) [19]. Qiu et al. proposed framework to quantify the potential gains of such techniques. Specifically, study the throughput performance gain that may be achieved by combining adaptive modulation and power control [20]. Kalyanaraman el al. examined scheduling algorithms to transmit multiple video streams from a base station to mobile clients. Authors present an epoch-by-epoch framework to fairly allocate wireless transmission slots to streaming videos [21]. Wong el al. presented algorithms for resource allocation in single carrier frequency division multiple access (SC-FDMA) systems, which is the uplink multiple access schemes considered in the Third Generation Partnership Project-Long Term Evolution (3GPP-LTE) standard [22].

\section{PROBLEM METHODOLOGY AND SYSTEM MODEL}

\subsection{Problem Methodology}

Among [13-22] discussed in related work discussed in section 2 4G HetNets, which employ OFDMA, downlink interference is practically reduced using RRM. This includes frequency spectrum allocation and power control, where in the case of interfering BSs, spectrum allocation minimises interference by allocating different subsets of subcarriers to those BSs. The most popular scheduling algorithms in OFDMA systems include MSR, MF, PRC, $\mathrm{PF}$ and the CDF based scheduling policy, where it retains a similar characteristic with PF scheduler that maximizes multiuser diversity and maximizes user's fairness. Due to this reason, PF based scheduler is commonly applied in the cellular environment. The best distributed RRM is the self- organizing approach which utilizes half of the available spectrum and adjusts the allocated subcarriers based on the interference received from the surrounding environment. A SON RRM based centralized technique which relies on the colored graph (CG) algorithm connecting node is visualized by the central processor if a SBS senses that at least one of its users is interfered. Basic CG SON RRM relies on each $\mathrm{BS}$ to measure the received power from surrounding BSs where the information of which neighboring. BSs are potentially interfering with its users is sent to the central node, which can be a Femto Management System (FMS). The SON RRM scheme uses a novel downlink interference mapping method in the form of a matrix of conflicts (MoC) to track how the BSs links affect certain UEs and locate possible interference instances per user.

\subsection{Proposed Scheme}

Here we propose a new techniquewith a hybrid algorithm called QoS aware optimal confederation (QoC). The QoC is based on radio resource management scheme for future generation networks, the individual base stations can take control of their allocated resource. The QoC-RRM introduces the hybrid recurrent deep neural network (RDNN) to classify the user in terms of priority and nonpriority based on multiple constraints. The chaotic weed optimization (CWO) algorithm used for QoC-RRM scheme. This algorithm proposed for routing to share queuing criterion information with neighbors.
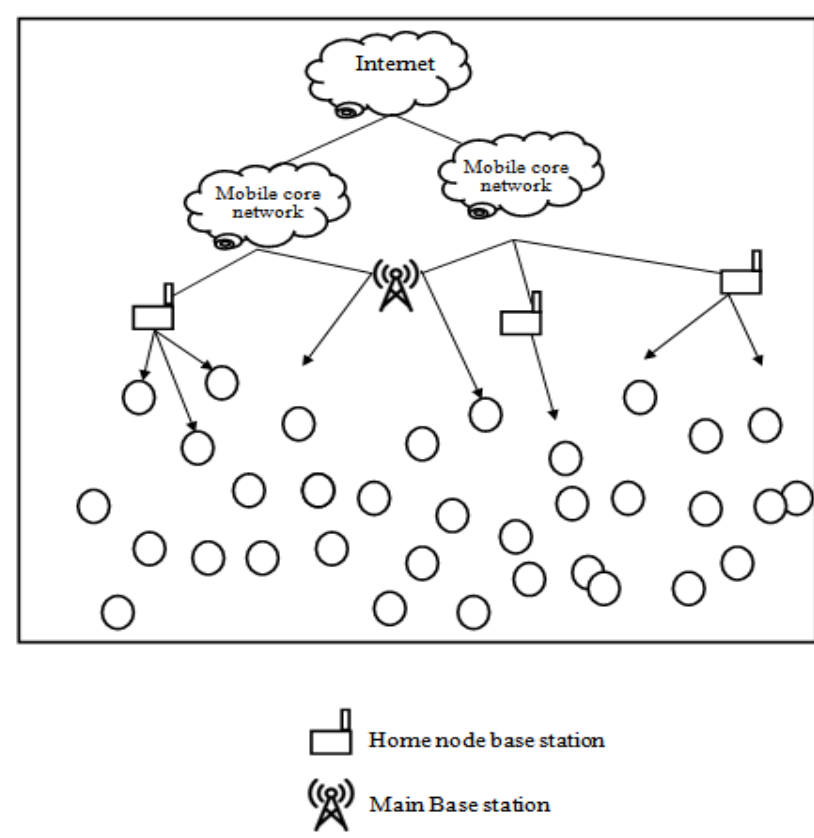

Fig. 1 System model of proposed QoC RRM

In parallel with the LTE radio access, packet core networks are also evolving to the flat SAE architecture. This new architecture is designed to optimize network performance, improve cost-efficiency and facilitate the uptake of massmarket IP based services. There are only two nodes in the SAE architecture user plane: the LTE base station (eNodeB) and the SAE Gateway, as shown in Figure 1.

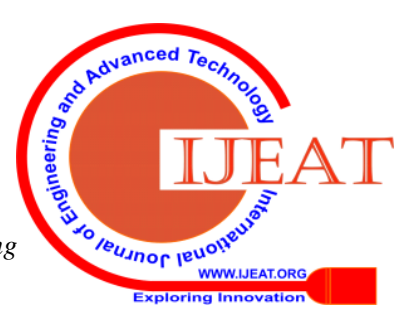


The LTE base stations are connected to the Core Network using the Core Network-RAN interface, S1. This flat architecture reduces the number of involved nodes in the connections.

\section{QOS AWARE OPTIMAL CONFEDERATION RADIO RESOURCE MANAGEMENT (QOC-RRM)}

\subsection{User classification using RDNN}

Recurrent neural systems or RNNs are a group of neural systems for handling consecutive data. Much as a convolutional system is a neural network that is specific for handling a matrix of values $X$, for example, a picture. A recurrent neural system is a neural system that is particular for preparing a succession of values $x^{(1)}, \ldots x^{(T)}$. To go from multilayer systems to recurrent systems, we have to exploit one of the early thoughts found in

machine learning and measurable models of the 1980s: sharing parameters crosswise over different parts of a model. Unfolding a recurrent computation into a computational diagram based on redundant structure, normally relating to a chain of occasions.

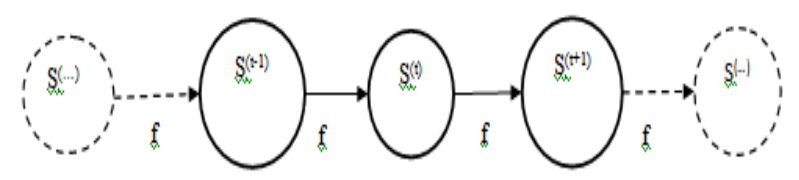

Fig. 2 The classical dynamical system

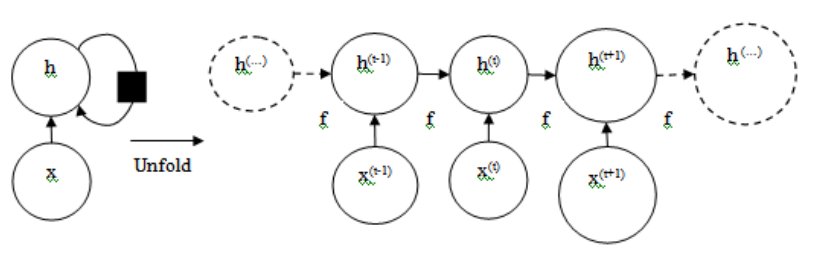

Fig. 3 Recurrent neural systems for user classification

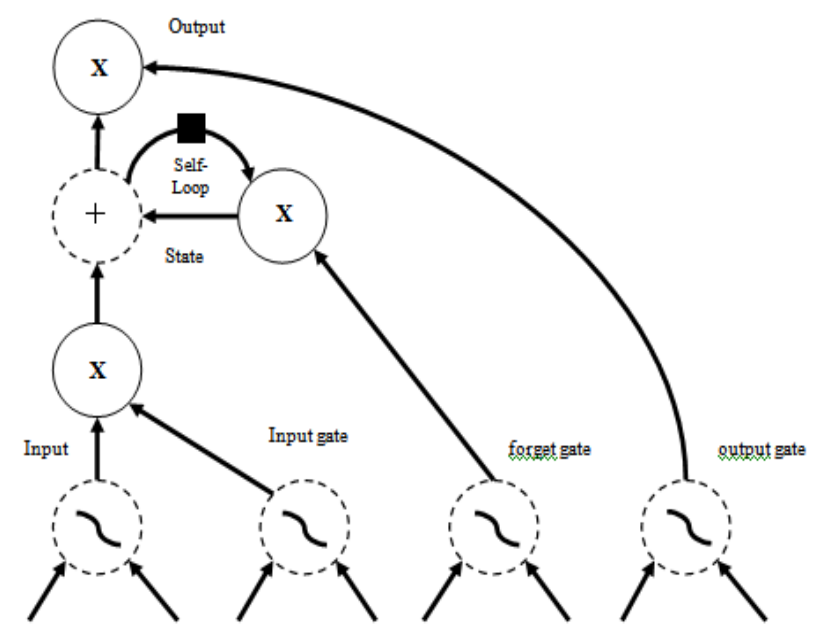

Fig.4 Block diagram of the LSTM recurrent network RNNs will include additional design highlights, for example, output layers that read data out of the state $h$ to make expectations. At the point when the recurrent system is prepared to play out an assignment that requires anticipating the future from an earlier time, the system commonly figures out how to utilize $h^{(t)}$ as a sort of lossy outline of the undertaking important parts of the past grouping of contributions up to $t$.

This rundown is all in all fundamentally lossy, since it maps a subjective length $\operatorname{succession}\left(x^{(t)}, x^{(t-1)}, \ldots, x^{(2)}, x^{(1)}\right)$ to a fixed length vector $h^{(t)}$. Contingent upon the preparation basis, this synopsis may specifically keep a few parts of the past succession with more exactness than different viewpoints. The mix of deep structures with worldly recurrent yields purported deep recurrent neural systems (DRNNs). In any case, this methodology ('back spread through time') experiences an evaporating or detonating inclination for larger $\mathrm{T}$, making the enhancement hard. Accordingly, RNNs are frequently not ready to beat DNNs in reasonable discourse preparing undertakings. One of the most established, yet still best arrangements proposed to cure this issue is to add structure to the RNN following the long shot term memory (LSTM) guideline as characterized.

$$
q_{i}^{(t)}=\sigma\left(b_{i}^{\circ}+\sum_{j} U_{i j}^{\circ} x_{j}^{(t)}+\sum_{j} W_{i j}^{\circ} h_{j}^{(t-1)}\right)
$$

\subsection{Route computation using chaotic weed optimization (CWO) method}

The chaotic weed optimization (CWO) algorithm is proposed in this paper for routing share queuing criterion data with other neighbors. The description of traditional CWO method of first process describes a randomly disperse the underlying seeds $s_{i}=\left(x_{1}, x_{2}, \ldots, x_{n}\right)$, where $n$ is the quantity of chosen variables, over the inquiry space. The next method describes the ability of every individual seed is determined by the improvement issue, and the seeds develop to weeds ready to create new units. The next third method of each individual is positioned dependent on its wellness concerning different weeds. In this way, each weed creates new seeds relying upon its position in the populace. The weeds which have gained more assets have a superior possibility of delivering seeds, and those which are less adjusted to the field are suspect to duplicate in this manner making less seeds.

$$
\text { Number of seeds }=\frac{F_{i}-F_{\text {warst }}}{F_{\text {best }}-F_{\text {worst }}}\left(N_{\max }-N_{\min }\right)+N_{\min }
$$

Here $F_{i}$ is the fitness of ith weed. $F_{\text {worst }}$, and $F_{\text {best }}$ represent the best and the worst capability in the weed population. 


\section{QoS Aware Optimal Confederation based Radio Resource Management Scheme for LTE Networks}

\section{RESULT AND DISCUSSION}

The performance of proposed QoC-RRM scheme for future generation network is implemented by using two different techniques called RDNN and CWO algorithm. The simulation will be focused on small cells network inside a $60 \mathrm{~m}$ by $60 \mathrm{~m}$ building close to an MBS equipped with two transmit antennas space-time-block-code (STBC) with antenna gain of $12 \mathrm{dBi}$.

The macro-cell Base stations (MBS) transmits power $2 \mathrm{~W}$ and $20 \mathrm{~W}$ of EIRP for its inner and outer users, respectively, while the SBS transmits $100 \mathrm{~mW}$ of EIRP and MBS has a height of $30 \mathrm{~m}$. In this simulation, macro cell user equipment's (UE) will be categorized as an inner user if the received signal for inner user signal transmission is higher than $20 \mathrm{~dB}$ above the noise power. Otherwise, this UE will be categorized as outer user. It will consider five HNBs to represent SBSs inside a building with two transmit antennas system and the capability to listen for MBS's signal. In addition, there are UEs inside the building as indoor users and UEs outside the building as outdoor users. HNBs and UEs are placed uniformly distributed within this building. Besides the considered UEs, there are 60 UEs registered as inner users and 20 UEs registered as outer users of the macro-cell with SINR uniformly distributed between $17 \mathrm{~dB}$ until $43 \mathrm{~dB}$ and $5 \mathrm{~dB}$ to $30 \mathrm{~dB}$, respectively.

The simulation parameters are summarized Below:

Table 1: Simulation parameters

\begin{tabular}{ll}
\hline Parameters & Values \\
\hline Network Size & $60 \times 60 \mathrm{~m}^{2}$ \\
Number of users & 30 \\
Gain of transmission antenna's & $12 \mathrm{dBi}$ \\
Receiver height & $2 \mathrm{~m}$ \\
Channel bandwidth & $10 \mathrm{MHz}$ \\
Initial energy of sensor nodes & $2 \mathrm{~J}$ \\
Transmission energy & $50 \mathrm{~nJ} / \mathrm{bits}$ \\
Receiving energy & $50 \mathrm{~nJ} / \mathrm{bits}$ \\
Data packet size & $500 \mathrm{bits}$ \\
MAC protocol & IEEE 802.11 \\
Bandwidth & $200 \mathrm{Kbps}$ \\
Simulation time & $100 \mathrm{~seconds}$ \\
\hline
\end{tabular}

3.1 Scenario 1: sum rate performance comparison

The proposed QoC-RRM scheme utilizes the user diversity improve the sum rate compared to the existing MOC based SONRRM allocation scheme. The proposed QoC-RRM provides the highest sum rate at various numbers of users.

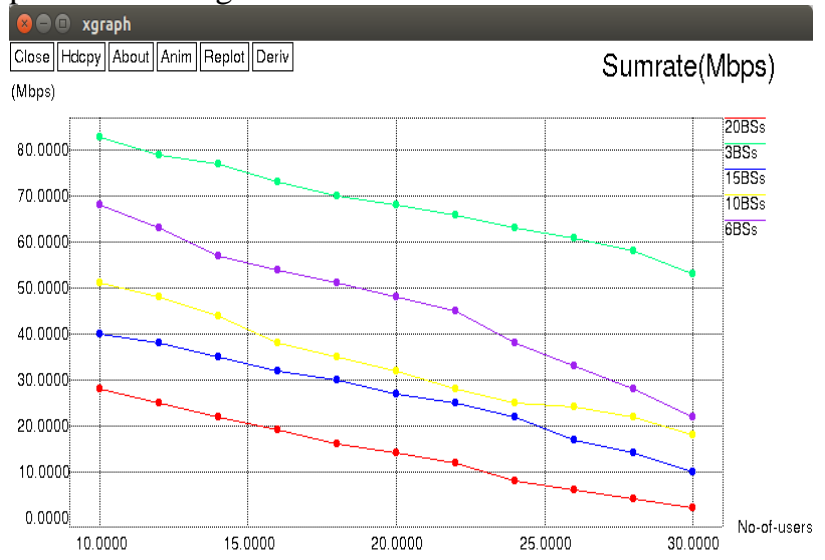

Fig. 5 Performance of sum rate with varying no. of users

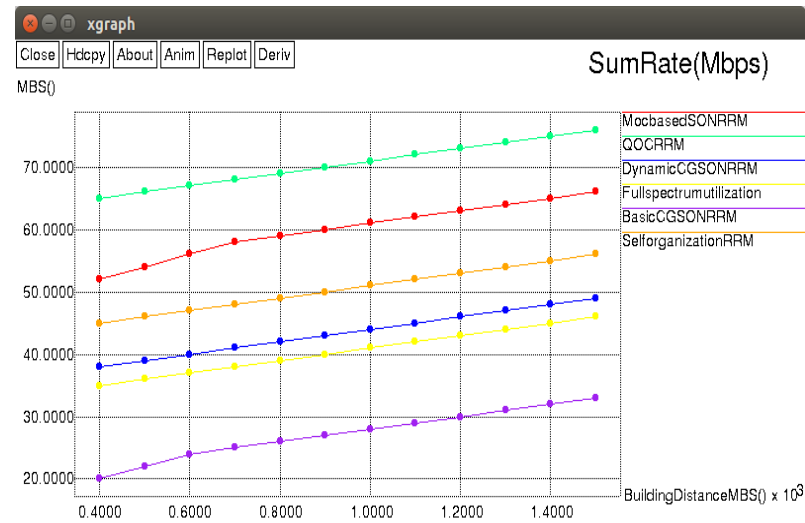

Figure 6. Performance of Indoor Sum rate

The figure 6 shows the Indoor performance of sum rate which gives the best sum rate compared with the different indoor users.

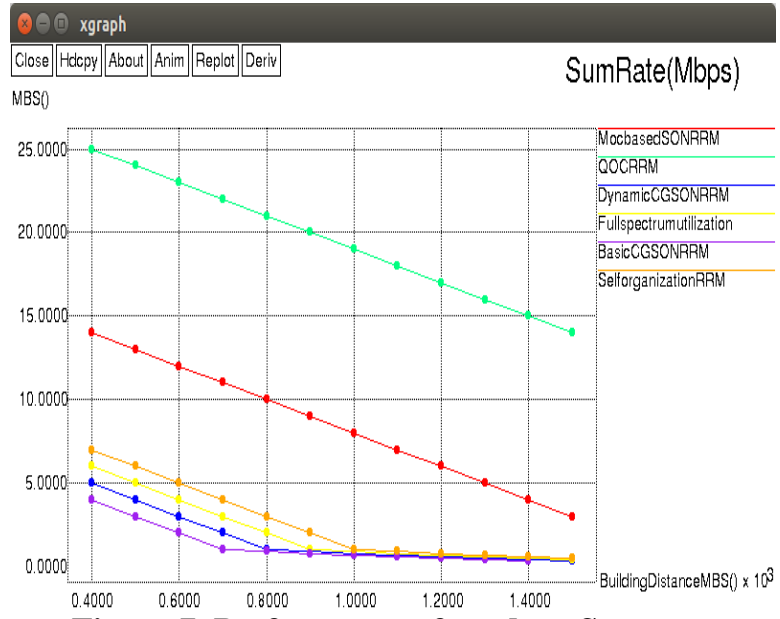

Figure 7. Performance of outdoor Sum rate

The figure 7 shows the outdoor performance of sum rate which gives the best sum rate compared with the different outdoor users.

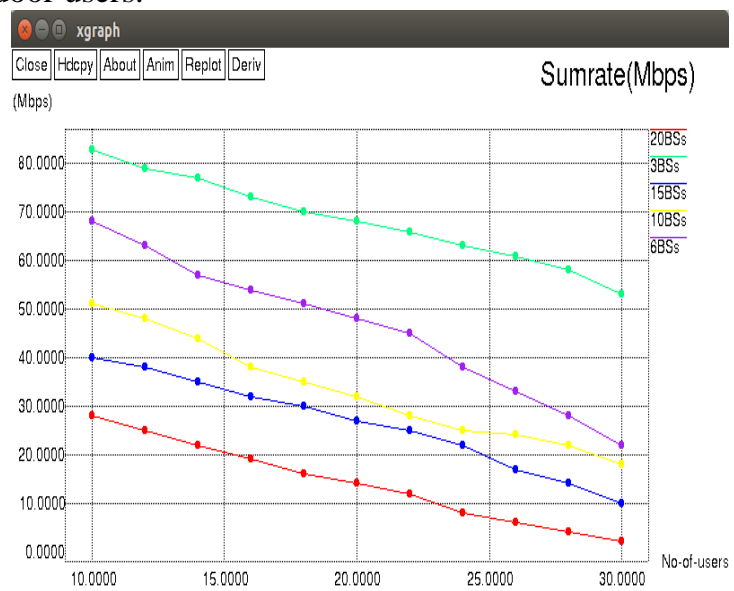

Figure 8. Performance of threshold Sum rate

The figure 8 shows the threshold performance of sum rate which has the best sum rate compared with the different base station. When no of user are increased the Mbps are maximum in our proposed threshold sum rate. Our proposed threshold sum rate use $3 \mathrm{BSs}$ which gives the best result compared to our existing system. 


\section{CONCLUSION}

We have proposed a QoS aware optimal confederation based radio resource management QOC-RRM method for Long Term Evolution (LTE) networks. In QOC-RRM scheme we described the hybrid recurrent deep neural network (RDNN) technique used to differentiate the operators by priority wise based on multiple constraints and it controlled the allocated resource by base stations. For routing share queuing criterion data with others chaotic weed optimization (CWO) algorithm are proposed and analyzed. Our QOC-RRM scheme is implemented with best result in Network Simulator (NS3) tool and performed with $10 \%$ better than conventional RRM schemes in terms of minimum date rate requirement, 50\% improved in QOCRRM compared with existing state-of-art schemes.

\section{REFERENCES}

1. Deb, S., \& Monogioudis, P. (2015). Learning-based uplink interference management in 4G LTE cellular systems. IEEE/ACM Transactions on Networking (TON), 23(2), 398-411.

2. Militano, L., Niyato, D., Condoluci, M., Araniti, G., Iera, A., \& Bisci, G. M. (2014). Radio resource management for grouporiented services in LTE-A. IEEE Transactions on Vehicular Technology, 64(8), 3725-3739.

3. El Essaili, A., Schroeder, D., Steinbach, E., Staehle, D., \& Shehada, M. (2014). QoE-based traffic and resource management for adaptive HTTP video delivery in LTE. IEEE Transactions on Circuits and Systems for Video Technology, 25(6), 988-1001.

4. Malandrino, F., Limani, Z., Casetti, C., \& Chiasserini, C. F. (2015). Interference-aware downlink and uplink resource allocation in HetNets with D2D support. IEEE Transactions on Wireless Communications, 14(5), 2729-2741.

5. Asheralieva, A., \& Miyanaga, Y. (2016). QoS-oriented mode, spectrum, and power allocation for D2D communication underlaying LTE-A network. IEEE transactions on vehicular technology, 65(12), 9787-9800.

6. Su, S. T., Huang, B. Y., Wang, C. Y., Yeh, C. W., \& Wei, H. Y (2016). Protocol design and game theoretic solutions for deviceto-device radio resource allocation. IEEE Transactions on Vehicular Technology, 66(5), 4271-4286.

7. Liu, J. S. (2018). Joint downlink resource allocation in LTEAdvanced heterogeneous networks. Computer Networks, 146, 85-103.

8. Liu, T. C., Wang, K., Ku, C. Y., \& Hsu, Y. H. (2016). QoSaware resource management for multimedia traffic report systems over LTE-A. Computer Networks, 94, 375-389.

9. Kong, C., \& Peng, I. H. (2017). Tradeoff design of radio resource scheduling for power and spectrum utilizations in LTE uplink systems. Journal of Network and Computer Applications, 78, 116-124.

10. Lucas-Estañ, M. C., \& Gozálvez, J. (2017). Distributed radio resource allocation for device-to-device communications underlaying cellular networks. Journal of Network and Computer Applications, 99, 120-130.

11. Jiang, M., Xenakis, D., Costanzo, S., Passas, N., \& Mahmoodi, T. (2017). Radio resource sharing as a service in 5G: a softwaredefined networking approach. Computer Communications, 107, 13-29.

12. Pramudito, W., \& Alsusa, E. (2014). Confederation based RRM with proportional fairness for soft frequency reuse LTE networks. IEEE Transactions on Wireless Communications, 13(3), 1703-1715.

13. Novlan, T. D., Ganti, R. K., Ghosh, A., \& Andrews, J. G. (2012). Analytical evaluation of fractional frequency reuse for heterogeneous cellular networks. IEEE Transactions on Communications, 60(7), 2029-2039.

14. Park, S., Seo, W., Kim, Y., Lim, S., \& Hong, D. (2010). Beam subset selection strategy for interference reduction in two-tier femtocell networks. IEEE Transactions on Wireless Communications, 9(11), 3440-3449.
15. Andrews, J. G. (2005). Interference cancellation for cellular systems: a contemporary overview. IEEE Wireless Communications, 12(2), 19-29.

16. Kim, J., \& Cho, D. H. (2010). A joint power and subchannel allocation scheme maximizing system capacity in indoor dense mobile communication systems. IEEE Transactions on Vehicular Technology, 59(9), 4340-4353.

17. Park, D., Seo, H., Kwon, H., \& Lee, B. G. (2005). Wireless packet scheduling based on the cumulative distribution function of user transmission rates. IEEE Transactions on Communications, 53(11), 1919-1929

18. Huang, Y., \& Rao, B. D. (2012). An analytical framework for heterogeneous partial feedback design in heterogeneous multicell OFDMA networks. IEEE Transactions on Signal Processing, 61(3), 753-769.

19. Chandrasekhar, V., \& Andrews, J. G. (2009). Spectrum allocation in tiered cellular networks. IEEE Transactions on Communications, 57(10), 3059-3068.

20. Qiu, X., \& Chawla, K. (1999). On the performance of adaptive modulation in cellular systems. IEEE transactions on Communications, 47(6), 884-895

21. Seetharam, A., Dutta, P., Arya, V., Kurose, J., Chetlur, M., \& Kalyanaraman, S. (2014). On managing quality of experience of multiple video streams in wireless networks. IEEE Transactions on Mobile Computing, 14(3), 619-631.

22. Wong, I. C., Oteri, O., \& McCoy, W. (2009). Optimal resource allocation in uplink SC-FDMA systems. IEEE Transactions on Wireless communications, 8(5), 2161-2165. 